Study, a follow-up study of patients who were diagnosed with cancer between 1970 and 1986 and who survived for at least five years. Of 6,068 female participants in the study who were eligible for analysis in the new study, 95 women developed 111 cases of breast cancer in adulthood. Details of the primary cancer diagnosis and treatment were compared between these women and the 5,973 participants who had not had breast cancer.

As expected, women who had received chest radiotherapy as children were at significantly increased risk of developing breast cancer. This applied to patients whose primary diagnosis was Hodgkin disease, bone or softtissue sarcoma, non-Hodgkin lymphoma or Wilms' tumor. In addition, breast cancer incidence was increased in patients who had not received chest radiotherapy but whose primary diagnosis was bone or soft-tissue sarcoma. Family history of breast cancer and personal history of thyroid disease were also revealed as risk factors for breast cancer, after adjusting for previous chest radiotherapy.

Kenney et al. recommend that the likelihood of secondary breast cancer should be assessed in all women who survive childhood cancer, and that early screening should be offered to those at increased risk.

Original article Kenney LB et al. (2004) Breast cancer after childhood cancer: a report from the Childhood Cancer Survivor Study. Ann Intern Med 141: 590-597

\section{p21 expression predicts efficacy of chemoradiotherapy for treatment of esophageal cancer}

Despite its toxicity, the associated increase in operative risk and variation in patient response, chemoradiotherapy (CRT) is regularly used in the treatment of esophageal cancer. Nakamura and colleagues investigated the association between response to CRT (and survival thereafter) and several cell-cycle regulators ( $p 53$, p21Waf1/Cip1, cyclin D1 and CDC25B). They demonstrated that tumoral expression of the cell-cycle regulator $\mathrm{p} 21$, is correlated with increased response to, and patient survival following, CRT.

The study comprised 76 patients with advanced esophageal cancer, who completed a planned CRT regimen. The response to treatment of primary and metastatic lesions was assessed on the basis of two-dimensional reduction rate, as seen by barium swallow X-rays, CT scans and endoscopy. In 32 patients who underwent esophagectomy, histological examination of the tissue was also used to classify response. Invasive squamous cell carcinoma samples from each subject were assayed for expression of the regulators by immunohistochemistry. Levels of p21 in pre-treatment biopsy specimens were closely correlated with response to CRT $(P=0.0001)$. Survival of patients with p21-positive tumors was significantly greater than those with p21-negative tumors $(P=0.013)$. The other cell cycle regulators assessed in this study did not affect treatment response or survival, although increased p21 expression was a significant predictor of survival in p53-negative tumors, but not p53-positive tumors.

The authors conclude that p21 expression is an independent variable which might predict the response of patients suffering from advanced esophageal cancer to CRT treatment, and their survival thereafter.

Original article Nakamura T et al. (2004) Expression of $\mathrm{p} 21^{\text {Wafl/Cip1 }}$ predicts response and survival of esophageal cancer patients treated by chemoradiotherapy. Dis Esophagus 17: $315-321$

\section{Surgical versus medical castration in prostate cancer patients}

Long-term treatment with luteinizing hormone releasing hormone $(\mathrm{LHRH})$ agonists has all but replaced surgical castration in patients with prostate cancer, partly because of the perceived advantage of testicular preservation. Some patients undergoing medical castration by this method have nevertheless reported significant decreases in the size of their testes. To investigate this, Issa and colleagues have compared testicular weight in men undergoing surgical castration with or without prior LHRH therapy.

This retrospective study included 88 men with prostate cancer, all of whom underwent bilateral simple orchiectomy with epididymal sparing. Prior to the procedure, 52 patients had been receiving $\mathrm{LHRH}$ agonist therapy (for a mean duration of 40.3 months) and the remaining 36 patients (the control group) had not. Following 